\title{
Radiation hybrid QTL mapping of Tdes2 involved in the first meiotic division of wheat
}

F. M. Bassi $1,2,5, \ldots *$ Dr S.F. Kianian is the corresponding author. F.M. Bassi is only the submitting author

Email: filippo.bassi@ndsu.edu
A. Kumar ${ }^{1,5}$
Q. Zhang 1,5

E. Paux $3, \ldots 55$ PLease add the following author after E. Paux and before E. Huttner:

M. Mergoum ${ }^{1}$

E. Huttner ${ }^{4}$
A. Kilian $^{4,5}$
R. Dizon ${ }^{1,5}$
C. Feuillet ${ }^{3,5}$
S. S. $X u^{4,5}$

S. F. Kianian ${ }^{1,5}$

Phone: +1-701-2317574

Fax:+1-701-2318474

Email: S.Kianian@ndsu.edu

${ }^{1}$ Department of Plant Sciences, North Dakota State University, Fargo, ND, 58102, USA.

${ }^{2}$ International Center for the Agricultural Research in the Dry Areas, Rabat, 10112, Morocco.

${ }^{3}$ Genetics Diversity and Ecophysiology of Cereals, INRA-UBP 1095, Clermont Ferrand, 63100, France. 
${ }^{4}$ Diversity Arrays Technology Pty Ltd., Yarralumla, ACT, 2600, Australia.

only Dr S. S. Xu is from '5' ARS Cereal Crops.. all other author are not

RS Cereal Crops Research

Unit, USDA, Fargo, ND, 58102, USA.

\section{Abstract}

Since the dawn of wheat cytogenetics, chromosome $3 \mathrm{~B}$ has been known to harbor a gene(s) that, when removed, causeds chromosome desynapsis and gametic sterility. The lack of natural genetic diversity for this gene(s) has prevented any attempt to fine map and further characterize it. Here, gamma radiation treatment was used to create artificial diversity for this locus. A total of 696 radiation hybrid lines were genotyped with a custom mini array of 140 DArT markers, selected to evenly span the whole $3 \mathrm{~B}$ chromosome. The resulting map spanned 2,852 centi Ray with a calculated resolution of $0.384 \mathrm{Mb}$. Phenotyping for the occurrence of meiotic desynapsis was conducted by measuring the level of gametic sterility as seeds produced per spikelet and pollen viability at booting. Composite interval mapping revealed a single QTL with LOD of 16.2 and $r^{2}$ of $25.6 \%$ between markers wmc326 and wPt-8983 on the long arm of chromosome $3 \mathrm{~B}$. By independent analysis, the location of the QTL was confirmed to be within the deletion bin 3BL7-0.63-1.00 and to correspond to a single gene located $\sim 1.4 \mathrm{Mb}$ away from $w p t$ 8983 Pt-8983. The meiotic behavior of lines lacking this gene was characterized cytogenetically to reveal striking similarities with mutants for the $d y$ locus, located on the syntenic chromosome 3 of maize. This represents the first example to date of employing radiation hybrids for QTL analysis. The success achieved by this approach provides an ideal starting point for the final cloning of this interesting gene involved in meiosis of cereals.

\section{Abbreviations}

3B-RH

Radiation hybrids for chromosome 3B

$\mathrm{cR}$

Centi rRays

CS

Chinese Spring

DArT

Diversity array technology

Gy

Gray

$H^{2}$

Broad sense heritability 


\section{LDN}

\section{Langdon}

LDN 3D (3B)

Langdon substitution line 3D for 3B

$\mathrm{M}_{1}$

MS

Mutant generation 1

Mean sum of squares

$\mathrm{MSg}$

Mean sum of squares for the genotype

MSge

Mean sum of squares for the genotype by environment

PV

Pollen viability

QTL

Quantitative trait loci

$\mathrm{RH}_{0}$ or $\mathrm{RH}_{1}$

Radiation hybrid generation 0 or 1

$\mathrm{SpS}$

Seeds per spikelet

SSR

Single sequence repeat

Communicated by P. Heslop-Harrison.

\section{Electronic supplementary material}

The online version of this article (doi:10.1007/s00122-013-2111-z) contains supplementary material, which is available to authorized users.

\section{Introduction}

Sexual reproduction is one of the most important processes in eukaryotes. The first meiotic division, in particular, has significant implications in plant evolution and breeding, since it is the stage during which recombination occurs. The synaptonemal complex is a tripartite proteinaceous structure that binds together the homologous chromosomes to stabilize the pairing interactions that take place between them, including crossing over (Hassold et al. 2009). Following DNA replication, the chromosomes in meiosis start condensing, and short stretches of axial elements are formed along their length (de Boer and Heyting 2006). These elements become clearly visible during leptotene and provide anchoring points for the installation of the central elements of the synaptonemal complex, which occurs at zygotene (Schramm et al. 2011). Recombination including crossing over takes place in the period from leptotene to zygotene (initially described in Henderson 1970; for review see Kim 2001). Then, the synapsed and condensed homeologs progress through diplotene to diakinesis, when the synaptonemal complex is gradually degraded leaving discrete bivalents linked to each other by chiasmata (Ross et al. 1996). 
The synaptonemal complex is essential for proper chromosome segregation in meiosis, but its role and necessity in favoring recombination remains unclear (Higgins et al. 2005; Qiao et al. 2012). Mutations affecting the correct formation of the synaptonemal complex will inevitably result in an increased yield of univalents at diakinesis, and ultimately in a reduction of male and female fertility. These types of mutants are defined as asynaptic, when no bivalents are formed, or desynaptic, when chromosomes form bivalents but then fell apart (as defined in Bourne and Danielli 1979; Cande and Freeling 2011; Murphy and Bass 2012). The first of this mutation was described in 1930 in maize (Zea mays L.) and named asynapticl (as1) (Beadle 1930). Wheat (Triticum aestivum L.) followed 15 years later with the discovery of the $d s$ genes (Li et al. 1945). Since then, many more meiotic mutants have been described in plants, including additional six in maize ( $d s y 1$ to $d s y 5$ and $d y 1$; for review see Cande and Freeling, 2011; http:/bivouac.cshl.edu/genewiki/index.php/Main_Page), 11 in rice (Oryza sativa L., $d s 1$ to $d s 11$; http://bivouac.cshl.edu/genewiki/index.php/Main_Page), at least three in Arabidopsis thaliana (asy1, dsy1, and dsy10; for review Caryl et al. 2003), and 15 in barley (Hordeum vulgare L., des 1 to des 15; Franckowiak and Lundqvist 2008). This is only a partial list, but it is evident that all plant species have a variable number of genes that when removed or mutagenized cause desynapsis.

In Triticum aestivum, a major gene involved in meiotic desynapsis was identified on chromosome 3B by Sears in 1954 while developing the aneuploidy stocks of the bread wheat variety 'Chinese Spring' (Sear 1954). Twenty years later, a different research group reached the same conclusion, finding a major desynaptic mutation on chromosome 3B of the Russian variety 'Avrora' (Zhirov et al. 1974). The existence of a durum wheat (Triticum turgidum ssp. durum (Desf.)-Husnot) gene with a similar mutant type was confirmed by Joppa and Williams (1988) while developing the variety 'Langdon' Dgenome substitution lines (Joppa and Williams 1993). Devos et al. (1999) further confined the location of this gene to the long arm of chromosome $3 \mathrm{~B}$, demonstrating that one dose of ditelosomic $3 \mathrm{BL}$ is sufficient to produce acceptable levels of fertility. This gene(s) does not have an official designation in wheat (McIntosh et al. 2011) and given its likely syntenic relationship to des 2 on barley chromosome 3H (Hernandez-Soriano and Ramage 1973), we propose the label of Tdes2, where 'des' stands for desynaptic and ' $T$ ' stands for Triticum.

The molecular characterization of this fertility-related gene(s) on 3BL has so far proven elusive. Despites being identified independently by three research groups and regardless of the fact that its chromosomal location has been known since the dawn of wheat cytogenetics, its lack of obvious genetic diversity has until now prevented any attempts at map-based cloning. One of the ways to move forward is to artificially create genetic diversity at this locus.

Martini and Bozzini (1966) successfully employed radiations to obtain desynaptic mutations in durum wheat. Radiation exposure damages the genome by causing random deletions (Baskaran 2010). When a deletion occurs in a gene (i.e. Tdes2), it often results in a knockout phenotype (Tanaka et al. 2010). In principle, there is no conceptual difference between the phenotypic change observed in the aneuploid stocks of wheat that have been used in the past (Gill et al. 1996; Faris and Gill 2003; Watanabe and Koval 2003), where a portion of the chromosome carrying the given locus was removed through the activity of a gametocidal gene (Gill et al. 1991), and a change due to the removal of the specific chromosome portion by radiation mutagenesis (Sutka et al. 1999; Raman et al. 2005). Indeed, the method adopted by Martini and Bozzini (1966) succeeded in generating recessive desynaptic mutations, but unfortunately those authors failed to exploit the genetic diversity they 
generated to pinpoint the specific gene(s) that had been mutagenized.

Radiation hybrid $(\mathrm{RH})$ is an experimental protocol also derived from radiation mutagenesis. It combines the DNA damage induced by mutagenesis with a hybridization step, to ultimately create mappable overlapping deletions that span the entire length of a chromosome (Goss and Harris 1975; Cox et al. 1990; see Faraut $\underline{2009}$ for review). This approach has been successfully adapted in wheat to generate high-resolution molecular maps of its chromosomes 1D, 3B and 5A (Hossain et al. 2004; Kalavacharla et al. 2006; Paux et al. 2008; Kumar et al. 2012a; Zhou et al. 2012; Michalak de Jimenez et al. 2013) and its entire D-genome (Kumar et al. 2012b; Tiwari et al. 2012). Among others, a great advantage of RH in wheat is that in vivo panels can be rapidly generated, allowing to combine the high-map resolution characteristic of this technique, with the ease of phenotyping for knockout mutations. Al-Kaff et al. (2008) successfully employed radiation knockout mutants to narrow down the genomic interval corresponding to the $P h 1$ locus, a gene controlling homologous pairing during meiosis in wheat. Here, it is described how the RH approach can be exploited to infer the genomic location of the Tdes2 gene(s) responsible for desynapsis in wheat, a gene lacking any known genetic diversity.

\section{Materials and methods}

\section{Plant material and RH population}

The present study used a RH mapping population developed for chromosome 3B (3B-RH panel) as described earlier (Paux et al. 2008; Kumar et al. 2012a). In brief, seeds of a durum wheat cultivar 'Langdon' (LDN) were irradiated at 250 and 350 Gray (Gy) doses of gamma ray and the resulting plants $\left(\mathrm{RH}_{0}\right.$ or $\left.\mathrm{M}_{1}\right)$ were crossed to LDN D-genome substitution line for chromosome 3B [LDN 3D (3B)]. The line LDN 3D (3B) has a pair of durum wheat 3B chromosomes replaced by a pair of 3D chromosomes transferred from 'Chinese Spring' hexaploid wheat (Joppa and Williams 1977, 1993). LDN 3D (3B) is male sterile, since the chromosome 3B contains a gene that is necessary to prevent desynapsis at meiosis. This line is maintained as disomic $3 \mathrm{D}$ and monosomic $3 \mathrm{~B}$. For the purpose of developing radiation hybrids, the plants nullisomic for $3 \mathrm{~B}$ were selected from the progeny of disomic 3D monosomic 3B, using markers specific for chromosome 3B. LDN 3D (3B) was used as female and crossed with $\mathrm{RH}_{0}$ plants to develop the $3 \mathrm{~B}-\mathrm{RH}$ panel. The seeds resulting from the cross are radiation hybrids generation $1\left(\mathrm{RH}_{1}\right)$, with chromosome $3 \mathrm{~B}$ and $3 \mathrm{D}$ in monosomic condition $\left(13^{\prime \prime}+3 \mathrm{~B}^{\prime 250} \mathrm{~Gy}+3 \mathrm{D}^{\prime}\right)$. The chromosome $3 \mathrm{~B}$ in these $\mathrm{RH}_{1} \mathrm{~s}$ carries deletion(s) in hemizygous (since the $3 \mathrm{~B}$ chromosome is monosomic) condition. A total of $696 \mathrm{RH}_{1}$ lines from the $3 \mathrm{~B}-\mathrm{RH}$ panel were used in the present study. In addition, normal (with no deletions) double monosomic (13" $+3 \mathrm{~B}^{\prime}+3 \mathrm{D}$ ) $\mathrm{F}_{1}$ lines were also generated by crossing LDN 3D (3B) with non-irradiated LDN and employed as experimental controls. The hexaploid wheat cultivar 'Chinese Spring' (CS) and four of its chromosome 3B deletion bin lines (Endo and Gill 1996) were also planted and used to locate the physical position of the DNA-markers.

All plants were grown under greenhouse controlled conditions (16-h light cycle; $15-25^{\circ} \mathrm{C}$; water, pesticide and fertilizer applications as required) at North Dakota State University (Fargo, ND; latitude: 46.8951, longitude: -96.8052 , elevation: $275 \mathrm{~m}$ ). 


\section{Phenotypic data on fertility/sterility}

Two methodologies were employed to determine the overall level of male fertility/sterility of each plant. The spikes from the first, third and fourth tillers were bag isolated at emergence and imposed to self-pollinate. After dehiscence, the spikes were harvested; for each spike, the number of spikelets and the number of seeds were counted. The level of fertility was determined for each spike as a ratio between the number of seeds produced and the total number of spikelets. The value of the three collected spikes was averaged to provide an overall 'seeds per spikelet' $(\mathrm{SpS})$ score. This value is rather provided in its unit value ranging from 0.0 to 1.41 , or as a percentage value of $\mathrm{SpS}$ fertility, where $100 \%$ correspond to the unit value 1.41 ( $\max$ value reached in the experiment). The second method used to measure fertility/sterility levels, took advantage of the emerging spike from the secondary tiller. This was collected always between 07:00 a.m. and 11:00 a.m. to minimize pollen dehydration, and then fixed in a 3:1 ethanol to acetic acid solution. After $48 \mathrm{~h}$, the fixed spikes were moved to a $70 \%$ ethanol solution and maintained at $4{ }^{\circ} \mathrm{C}$ until analysis. For each spike, three anthers from different spikeletes were then dissected and stained on a microscope slide with a non-toxic dye described in Peterson et al. (2010). The number of live and dead pollen grains was counted by observing the stained pollen (Fig. 1) under a 20× magnification optical microscope (Zeiss, Germany). A minimum of 200 pollen grains were counted and the overall pollen viability (PV) score was calculated as the percentage of non-aborted pollen grains over the total number of pollen grains. The data on fertility were recorded for each $\mathrm{RH}_{1}$ plant as well as for three durum control lines (LDN, LDN 3D (3B), and their $\mathrm{F}_{1}$ progeny) and two hexaploid control lines (CS and the CS deletion bin line 3BL7-0.63-1.00). The $\mathrm{RH}_{1}$ lines, due to their unique karyotype makeup, could only be tested once during winter 2009 without replicates by single plant screening. The control lines, however, were tested in three replicates across four greenhouse seasons in Fargo using a randomized complete block design: winter 2009, spring 2010, winter 2011, and spring 2011; while the hexaploid controls were tested in three replicates solely during the winter 2011 season.

Fig. 1

Physiological effects of removing the 3B chromosome from the genome of durum wheat. a An adult wheat spike opening its florets to facilitate crosspollination, because the sterility of the pollen grains prevents self-pollination. b Optical microscope imaging $(\times 20)$ of mature pollen grains, where the sterile pollen grain results from abnormal meiosis 


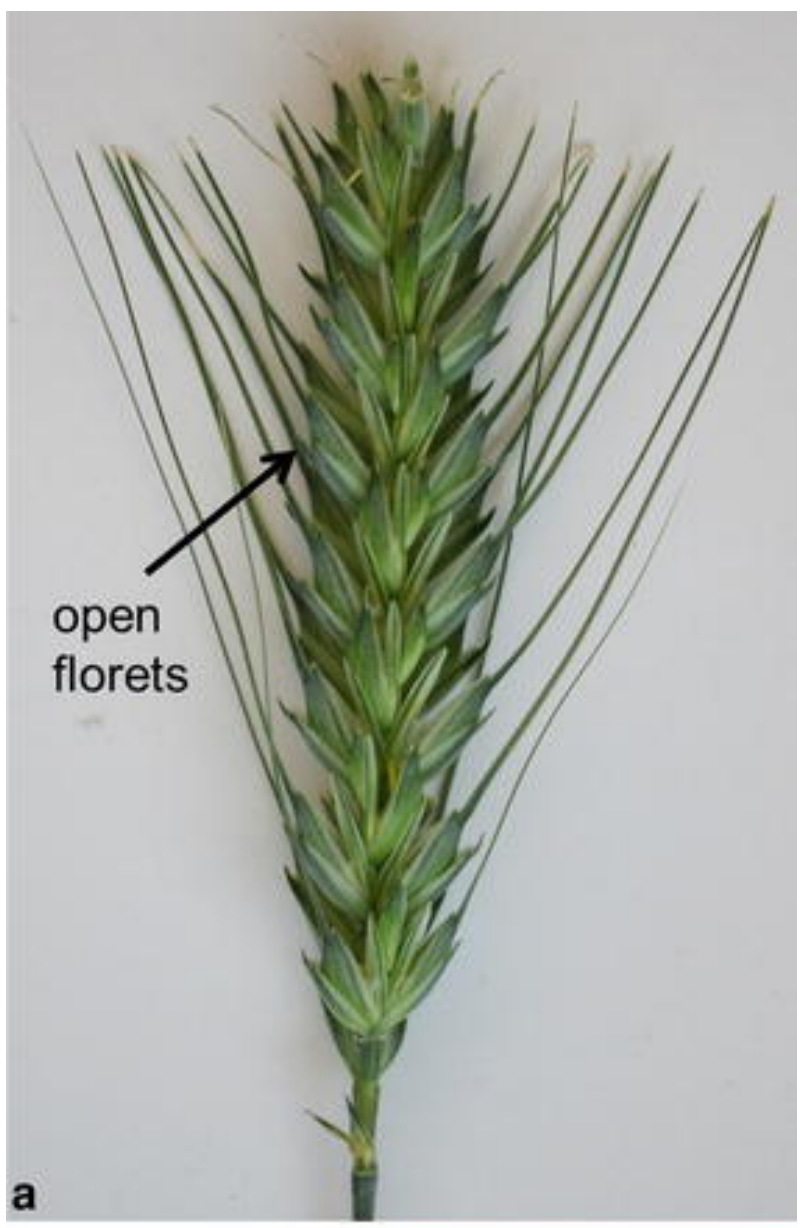

vital

pollen

\section{aborted}

pollen

b

Cytological studies of the defective meiosis were conducted on immature spikes of LDN 3D (3B) collected 3-5 days before heading (Zadok scale stage 41-45). Young spikes were fixed in a 3:1 mixture of ethanol and acetic acid for $48 \mathrm{~h}$ at room temperature and then maintained as described for PV phenotyping. Anthers were transferred to a glass slide with the aid of a dissecting microscope. The pollen mother cells were squeezed out of the anthers in Carbol fuchsin stain (Xu and Joppa 1995) with the use of a scalpel, and then were squashed under a cover glass. The slides were examined for pollen mother cells undergoing meiotic division using a Zeiss Axioplan 2 Imaging Research Microscope (Carl Zeiss Light Microscopy, Germany). The images of pollen mother cells at the stages from diakinesis to tetrad were captured using an Axiocam HRm CCD camera and imaging software Aixovision Release 4.5 (Carl Zeiss Light Microscopy, Germany). In aberrant cells, the number of 
bivalents can be measured as ( 28 - number of univalents) $/ 2$.

\section{Molecular analysis}

DNA from each genotype was extracted from lyophilized leaf tissue of 1 month-old plants as described by Guidet et al. (1991). In the case of $\mathrm{RH}_{1}$ lines, the leaves were collected from the same individuals that were employed for phenotypic evaluations. All DNA samples were equilibrated to a concentration of $50 \mathrm{ng} / \mu \mathrm{l}$ using the NanoDrop spectrophotometer (Thermo Scientific, DE). The integrity and quality of the DNA were tested by placing $4 \mu \mathrm{l}$ of extract in a $1 \times$ restriction enzyme buffer (without the actual restriction enzyme) at $35^{\circ} \mathrm{C}$ for $6 \mathrm{~h}$, followed by separation on a $1 \%$ nondenaturing agarose gel, and visual evaluation after staining with GelRed (Biotium Inc., CA). The restriction buffer stimulates the activity of any DNAse present in the extract, hence promoting DNA degradation. High-quality extracts show a single sharp band in close proximity of the gel wells and such extracts were used for further genotyping.

The 3B-RH panel was genotyped using three different classes of markers: PCR-based insertion sitebased polymorphisms (ISBPs, labeled as 'cfp'; Paux et al. 2006, 2010); simple sequence repeats (SSRs, labeled as 'gwm' and 'wmc'; Somers et al. 2004; Song et al. 2005) and Diversity Array Technology (DArT, labeled as 'wPt' and 'tPt'; Akbari et al. 2006; Wenzl et al. 2010). The SSR and ISBP markers were primarily used to confirm the genotyping data provided by the DArT markers. PCR amplification and scoring of ISBP and SSR markers were done as described in Kumar et al. (2012a). For DArT analysis, a total of 245 3B specific DArT markers were selected from earlier studies (175 markers; Wenzl et al. 2010; Huang et al. 2012) and the RH map presented in Kumar et al. (2012a). These markers were selected to represent the whole length of chromosome 3B. A mini custom array containing 490 markers was generated, with each of the 245 selected DArT clones blotted in duplicate. All $696 \mathrm{RH}_{1}$ lines, four chromosome 3B deletion bin lines, positive control $\left(\mathrm{F}_{1}\right.$ : $13^{\prime \prime}+3 \mathrm{~B}^{\prime}+3 \mathrm{D}^{\prime}$; eight replicates) and negative control [LDN 3D (3B): $13^{\prime \prime}+3 \mathrm{~B}^{0}+3 \mathrm{D}^{\prime \prime}$; eight replicates] were hybridized to the custom array following the provider protocol (Akbari et al. 2006; Wenzl et al. 2010). Positive hybridization was scored as 'retention' and lack of hybridization was scored as 'deletion'. Only those markers that resulted in identical scoring between the two duplicates, and which hybridized only to the positive control but not to the negative, were accepted and used for the construction of the RH map of chromosome 3B. The retention frequency of an individual measures the portion of markers retained over the total number of markers genotype, and is an inverse indicator of the amount of radiation damage sustained by a given plant (i.e. 0.99 retention frequency means that only $1 \%$ of the markers identified a deletion, while 0.80 means that $20 \%$ of the marker loci had been deleted; Kumar et al. 2012a).

\section{Statistical analyses}

To generate a comprehensive map of chromosome 3B, the retention/deletion data on the $696 \mathrm{RH}_{1}$ lines genotyped with 140 markers were run on the Carthagene mapping software v1.2.2 (de Givry et al. 2005), employing the iterative framework mapping procedure described earlier (Kumar et al. 2012a). Further details are provided as supplementary material. The calculation of the map resolution provided by this population was computed as suggested by Kumar et al. (2012a). The SAS 9.3 environment (SAS Institute, Cary, NC) was used to determine the significant differences of the fertility 
categories ( $t$ test) by procGLM across four growing seasons and then combining (12 replicates) given the absence of statistical significance between seasons. SAS 9.3 was also employed to calculate the $r^{2}$ association between markers and phenotypes. Phenotypic data on fertility and RH mapping data were used to conduct QTL analysis using composite interval mapping (CIM) by Window QTL Cartographer V2.5 employing the default cofactors (model 6, window size $10 \mathrm{cR}$, backward regression method, 5 control markers; Wang et al. 2011). The threshold LOD score for detection of definitive QTL was calculated based on 1,000 permutations (Churchill and Doerge 1994). Only $\mathrm{RH}_{1}$ lines with retention frequency smaller than 0.999 and higher than 0.001 were used for the QTL analysis. Single marker regression was also employed to confirm the results and to identify the closely linked marker loci.

Broad sense heritability $\left(H^{2}\right)$ was estimated using the formula:

$$
1-\frac{\text { MSge }}{\mathrm{MSg}}
$$

which uses the mean sum of squares (MS) of the genotype (MSg) and genotype by environment (MSge) (Falconer and Mackay 1996; Tsilo et al. 2011).

\section{Results}

\section{A custom DArT array for chromosome 3B}

Based on genetic (Wenzl et al. 2010; Huang et al. 2012) and RH (Kumar et al. 2012a) DArT maps available, 245 DArT clones were selected to evenly cover the length of chromosome $3 \mathrm{~B}$. Each clone was blotted in duplicate on a mini custom array, and a total of $136(55 \%)$ always showed hybridization with the positive control DNA (containing chromosome 3B) and no hybridization with negative control DNA (lacking chromosome 3B). The hybridization test was performed on 16 parental biological replicates (eight positive and eight negative) with each marker scored for both duplicates. The markers that were excluded rather did not prove consistent across replicates (31\%) or did not hybridize on LDN because specific for bread wheat (24\%). To map these DArT markers to specific deletion bins, the custom array was also hybridized to CS and four of its chromosome $3 \mathrm{~B}$ deletion bin lines. Twenty-four markers (18\%) hybridized to the CS DNA and could be precisely localized to one of the five chromosomal portions determined by the four deletion bin lines.

\section{Characterization of a large 3B-RH population}

The whole population of $6963 \mathrm{~B}-\mathrm{RH}_{1}$ lines was characterized using 136 DArT and four PCR markers. Based on 140 markers data, the average retention frequency across the whole 3B-RH panel was 0.80 (Fig. 2). However, 233 (33\%) of the lines had no detectable deletions or missed the entire chromosome 3B (133 lines, retention frequency $=1.00 ; 100$ lines, retention frequency $=0.00)$. Thus, these RH lines were excluded from further analysis because these did not provide any exploitable mapping information. A total of 463 (67\%) lines showed deletions; $56 \%$ had a marker loss for chromosome $3 \mathrm{~B}$ between 1 and $10 \%$, while $11 \%$ of the lines had more than $10 \%$ of the chromosome deleted, with seven 3B-RH lines carrying less than $50 \%$ of the chromosome (Fig. 2 ). 
Overall the retention frequency of these segregating lines was 0.93 .

Fig. 2

Distribution of retention frequencies in a population of 696 radiation hybrid lines. The majority of the lines have $1-10 \%$ of chromosome 3B deleted

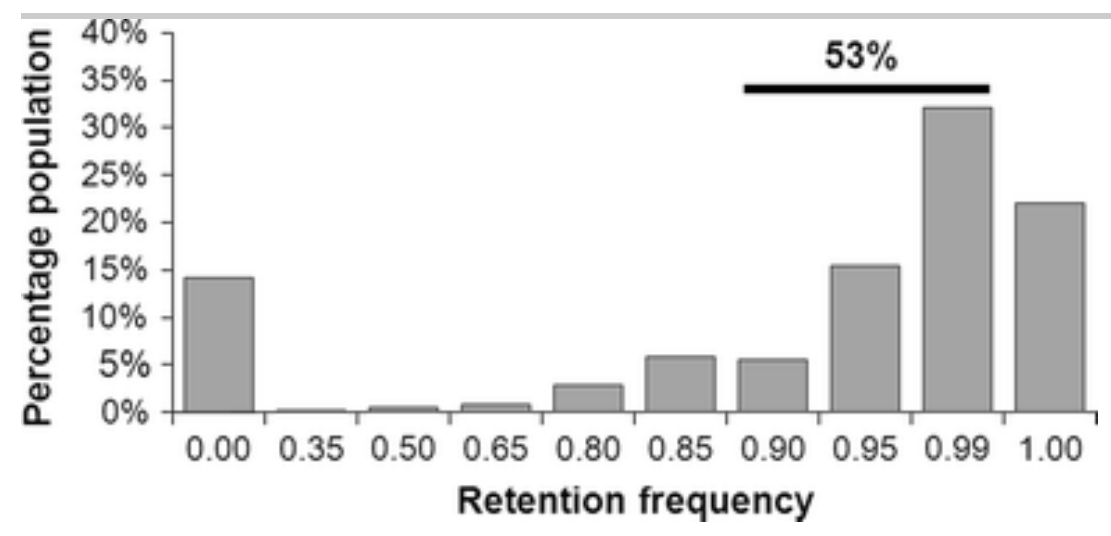

\section{A radiation hybrid map for chromosome 3B}

The scoring data of 140 good quality markers on 463 informative $\mathrm{RH}_{1}$ lines were used to generate a framework RH map of chromosome 3B. All the markers formed a single group at LOD score of 70.0 and distance of $50.0 \mathrm{cR}$. The markers order was maintained as predicted in the previous 3B-RH map (Kumar et al. 2012a), while the markers that had not been previously mapped were positioned using an iterative framework mapping approach (see supplementary material for details). The 28 markers (24 DArTs and 4 PCR-based) for which the bin location was known, respected their bin positioning in the final 3B-RH map, de facto sectioning the 3B chromosome into five large physical portions: $3 \mathrm{BS}$ telomere (3BS3-0.87-1.00), 3BS arm (3BS8-0.78-0.87), 3BS pericentromere (3BS1-0.33-0.55), centromere plus 3BL pericentromere, and the 3BL arm plus telomere (3BL7-0.63-1.00) (Fig. 3 ). The final 3B-RH map generated with 140 markers spans a total distance of 2,852 cR. The average marker density is one marker every $20 \mathrm{cR}$, with a standard deviation of $8.3 \mathrm{cR}$; in fact, $102(73 \%)$ markers are spaced $20 \pm 8.4 \mathrm{cR}$, while just $20(14 \%)$ markers are at a distance superior to $28.4 \mathrm{cR}$ with a maximum of $40.5 \mathrm{cR}$, and $18(13 \%)$ at a distance inferior to 11.6 with a minimum of $1.6 \mathrm{cR}$. The even markers distribution confirms that the strategy adopted to select the DArT clones for the custom array provided uniform coverage of the $3 \mathrm{~B}$ chromosome. This chromosome is $993 \mathrm{Mb}$ in size (Dolezel et al. 2009), suggesting a map resolution of $0.348 \mathrm{Mb} \mathrm{cR}^{-1}$ and an average physical distance of $\sim 7.0 \mathrm{Mb}$ between two marker loci.

Fig. 3

Radiation hybrid (RH) map of chromosome 3B short and long arms based on 140 markers loci and 696 RH lines. Map distances are rep orted in $\mathrm{cR}$. The chromosome is divided into deletion bins (solid vertical line) on the basis of known marker position; the remaining chromosomal locations (dashed vertical line), including the centromere and pericentromere, are derived on the basis of map positions. The location of the fertility-related Tdes 2 locus is presented as significance of single marker regression analy sis, each star represent a decimal of significance bey ond p:0.0001 (i.e. **** indicates $p: 0.0000001$ ) 


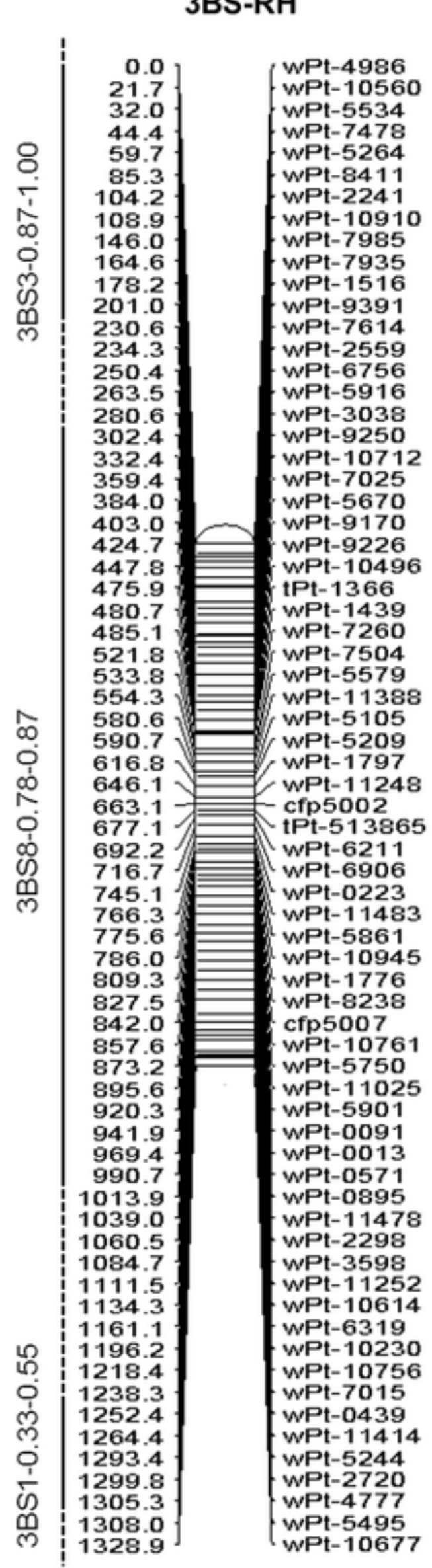

3BL-RH

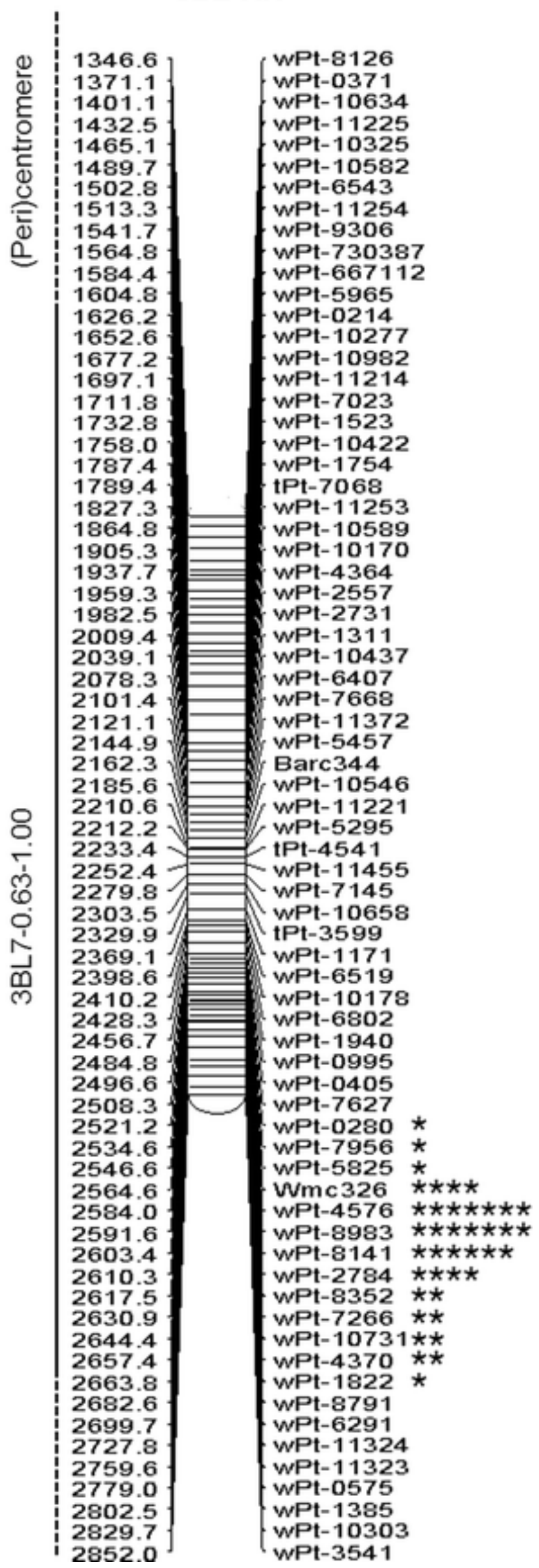




\section{The 3B radiation hybrid population segregates for fertility}

Chromosome $3 \mathrm{~B}$ is known to carry a gene that controls chromosome synapsis at meiosis that, when mutagenized, causes improper chromosome segregation and ultimately loss of fertility (Sears 1954; Joppa and Williams 1988; Devos et al. 1999). The level of fertility is then a good indicator of chromosomes behavior at meiosis. In this study, three genotypes carrying two, one, and zero doses of chromosome 3B (and the inverse number of chromosome 3D doses) were examined for their level of fertility (Table 1). The interaction between growing seasons (four seasons in one location) and genotype was not significant, while genotype segregation was significant at 0.001 level of confidence. The number of seeds produced by each spikelet drastically decreased when reducing the dosage of chromosome $3 \mathrm{~B}$, being 1.41 in the normal disomic, 0.39 in the hemizygous and 0.08 in nullisomic conditions. The LDN 3D (3B) line produces zero to one seed per spike, showing the characteristic open florets phenotype (Fig. 1) indicative of strong male sterility. Further, the pollen analysis (Fig. 1) showed that normal LDN lines (containing both copies of chromosome 3B) have a pollen viability of $80 \%$, while it was $60 \%$ in the $\mathrm{F}_{1}$ (containing a single copy of chromosome $3 \mathrm{~B}$ ) and only $23 \%$ in LDN 3D (3B) (entirely missing chromosome 3B) (Table 1 ). Thus, using both seed set (SpS) and pollen viability (PV) allowed the identification of three classes of fertility based on the dosages of chromosome 3B (Table 1). Further, the CS deletion line 3BL7-0.63-1.00, deficient for the telomeric portion of 3BL, analyzed for SpS and PV showed sterility levels non-significantly different from the line missing the entire 3B chromosome. The broad sense heritability was extremely high (0.98) for both traits, but it must be pointed out that this heritability is calculated on the segregation of chromosome $3 \mathrm{~B}$ rather than single genes, and that the test environments were greenhouse seasons, where the environmental effects are expected to be minimized. Still, the use of these controlled environments to test for the two fertility traits should allow to properly measure the genetic effect as indicated by the high $H^{2}$.

Table 1

Variations of fertility at different dosages of chromosome 3B

\begin{tabular}{|l|l|l|l|}
\hline Karyotype & Seeds per spikelet & Pollen viability & Fertility class \\
\hline $13^{\prime \prime}+3 \mathrm{~B}^{\prime \prime}+3 \mathrm{D}^{0}$ & $1.41 \mathrm{a}$ & $0.80 \mathrm{a}$ & Fully fertile \\
\hline $13^{\prime \prime}+3 \mathrm{~B}^{\prime}+3 \mathrm{D}^{\prime}$ & $0.39 \mathrm{~b}$ & $0.60 \mathrm{~b}$ & Partially fertile \\
\hline $13^{\prime \prime}+3 \mathrm{~B}^{0}+3 \mathrm{D}^{\prime \prime}$ & $0.08 \mathrm{c}$ & $0.23 \mathrm{c}$ & Sterile \\
\hline $20^{\prime \prime}+3 \mathrm{BL}^{\prime}-0.63-1.00^{\prime \prime} \mathrm{B}$ & $0.07 \mathrm{c}$ & $0.18 \mathrm{c}$ & Sterile \\
\hline LSD & & 0.07 & \\
\hline
\end{tabular}


", disomic; ', monosomic; ${ }^{0}$, nullisomic; $\mathrm{H}^{2}$, broad sense heritability

A Average of 12 replicates ( 3 replicates across four seasons)

${ }^{B}$ Average of three replicates collected in one season

The $3 \mathrm{~B}-\mathrm{RH}_{1}$ lines have the chromosome $3 \mathrm{~B}$ in monosomic state, which might or might not carry deletions as consequence of the radiation treatment. Thus, $3 \mathrm{~B}-\mathrm{RH}_{1}$ monosomic lines are expected to have moderate levels of fertility similar to the $\mathrm{F}_{1}$ monosomic plant; this level of sterility should drop to the nullisomic state of LDN 3D (3B) when the Tdes2 gene(s) responsible to provide fertility is lost through radiation damage. Six hundred and forty-six 3B-RH lines were phenotyped for $\mathrm{SpS}$ (50 lines never reached flowering), and those lines that had a value of $\mathrm{SpS}$ falling in between two fertility classes (Table 1) were scrutinized under the microscope to measure PV. A total of 376 lines were characterized for $\mathrm{PV}$. Unfortunately, $\mathrm{RH}_{1}$ lines are unique genotypes which segregate in a nonMendelian fashion in the following filial generations; hence, only single plant measurements without replication could be performed. However, in order to increase the accuracy, multiple measurements (sampling) were obtained from each $\mathrm{RH}_{1}$ individual. Also, an overall fertility value (' 1 ' for fertile and ' 0 ' for sterile) was given only when the value of $\mathrm{SpS}$ clearly fell into one of the fertility classes (Table 1) for all the sampling/measurements collected. When both SpS and PV values were collected, an overall fertility value was given only to those lines showing good agreement between the two phenotype scorings. The values for these two traits for all lines were converted into a percentage of fertility as compared to the most fertile $3 \mathrm{~B}-\mathrm{RH}$ plant $(\mathrm{SpS}=1.41$ and $\mathrm{PV}=0.9)$ and are presented in Fig. 4. Overall, for 616 lines ( $89 \%$ ), it was possible to determine a unique value for fertility (rather a clearly determined SpS, or both SpS and PV), 123 being sterile (20\%) and 493 fertile (80 \%). Of these phenotyped lines, $416 \mathrm{RH}_{1}$ also segregated for chromosome $3 \mathrm{~B}$ at the genetic level (i.e. retention frequency between 0.01 and 0.99 ), with 42 sterile lines (10\%) and 374 fertile (90\%). In addition, 47 lines segregate for chromosome $3 \mathrm{~B}$ but do not fall within any of the fertility classes and were then excluded from the QTL analysis.

Fig. 4

Segregation among 696 radiation hybrid lines for two fertility-related traits. Both traits are presented as percentage of maximum fertility as compared to a fully fertile control line. The four motions polynomial trend lines separate the values into two classes, also marked by gradient of gray in the background. Lines falling within the lighter gray area (30-55 \%) had a fertility state that could not be precisely determined 


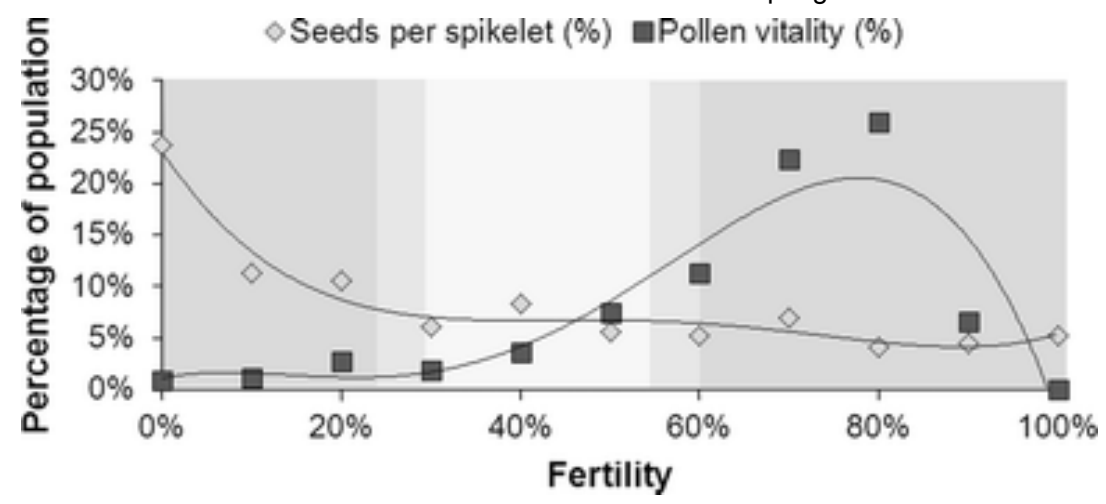

\section{Mapping fertility-related gene Tdes2}

Association between the 140 marker genotypes and the overall fertility value of the $\mathrm{RH}_{1}$ lines was investigated using QTL analysis. Those lines without any detectable deletions or lacking the entire 3B chromosome were excluded from the QTL analysis because it would identify a positive association at every marker locus. Composite interval mapping of the 416 informative 3B- $\mathrm{RH}_{1}$ lines identified a single QTL, named QTdes2.ndsu-3B (following guidelines by McIntosh et al. 2011), with a LOD score of 16.2 and a $r^{2}$ of $25.6 \%$ (Fig. $\underline{5}$ ). The threshold LOD score based on 1,000 permutations was 6.9. This QTL is flanked by the marker loci $w m c 326$ and $w P t-8141$, spanning a $38.8 \mathrm{cR}$ region $(2,564.6-2,603.4 \mathrm{cR})$. Based on the overall resolution of this map $(1 \mathrm{cR}=0.348 \mathrm{Mb})$, the QTL interval of $38.8 \mathrm{cR}$ corresponds to an approximate physical size of $13 \mathrm{Mb}$. To further confirm the results, all 140 mapped loci were searched for association with the overall fertility score using single marker regression. A significant association $\left(p=1 * 10^{-8}\right)$ was observed for markers $w P t-4576$ and $w P t-8983$ which were located at positions 2584.0 and $2591.6 \mathrm{cR}$, respectively, on the RH map. These two markers are mapped within the markers interval wmc326 and $w P t-8141$ where CIM identified the major QTL, thus, confirming the results of CIM. These markers are located inside the 3BL7-0.63-1.00 deletion bin (Fig. 3). Marker $w P t-8983$ has a retention frequency of 0.93 among the 416 segregating lines; it is deleted in 18 of the sterile lines (42\%) and retained in 347 of the fertile (93\%), for a total of 365 (92\%) of the lines with genotyping and phenotyping perfectly matching.

Fig. 5

Location of the Tdes 2 fertility-related QTL on the chromosome 3B radiation hybrid map. Association between fertility status and genotyping data of 416 radiation hybrid lines was computed by composite interval mapping. The LOD values are reported in the black line (left $Y$ axis) and the $r^{2}$ values in the gray line (right $Y$ axis). The dashed line indicates the threshold significance value calculated with 1,000 permutations. The significant marker loci are reported with their LOD values in parenthesis 


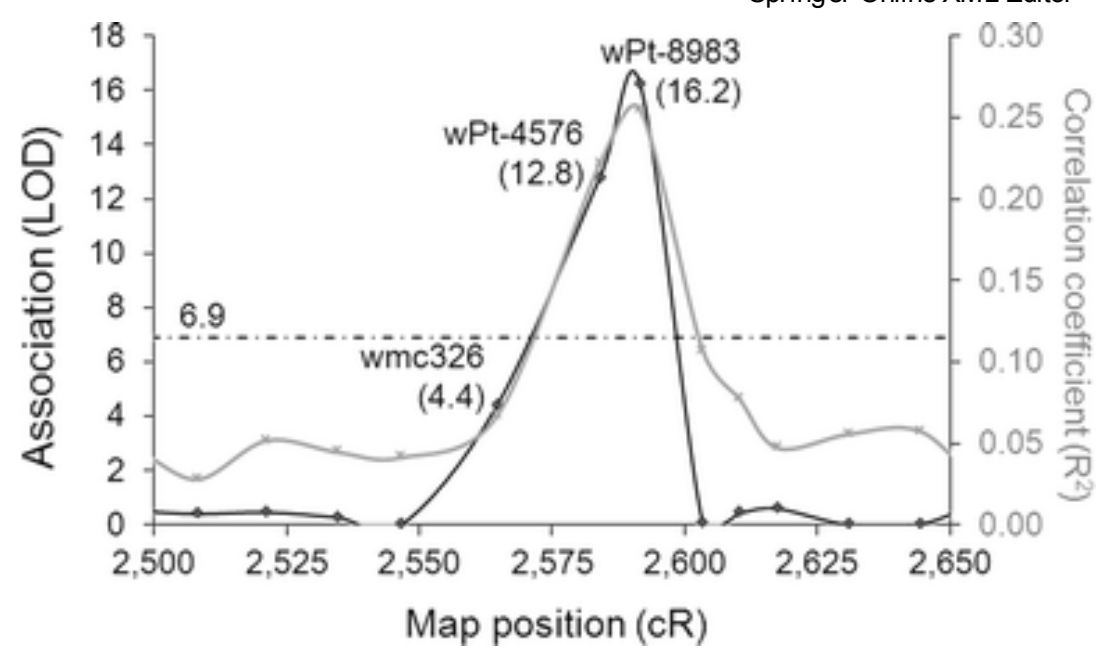

\section{The desynaptic nature of the $3 B$ fertility gene}

The substitution line LDN 3D (3B) lacks the entire 3B chromosome, including the Tdes2 locus. As a result, it suffers from drastic male sterility (Table 1 ) and partial female fertility (Joppa and Williams 1988). Cytogenetic analysis revealed that the pollen mother cells have an abundance of univalents at metaphase I (Fig. $\underline{6} a$ ), lagged chromosomes at anaphase I (Fig. $\underline{6} b$ ), bridge structures at telophase I (Fig. $\underline{6}$ c), and abundant micronuclei at tetrad stage (Fig. $\underline{6}$ d). These are typical cytological characteristics of desynapsis mutations. In the course of this study, the number of univalents observed at late diakinesis/early metaphase was not recorded as previous studies had already reported on it (Joppa and Williams 1988; Xu and Joppa 2000) and the primary target of this study was to observe the desynaptic nature of Tdes 2 mutants, rather than confirming its nature. However, in the pictures that were taken, the number of univalents ranged from 4 to 16 (or 2 to 8 bivalents). In over 20 pollen mother cells staged at metaphase I, it was never possible to observe complete lack of bivalents.

Fig. 6

Cytological characterization of pollen mother cells lacking chromosome 3B ( $\Delta T$ des 2$)$. a A cell with seven bivalents and 14 univalents at metaphase I. b A cell with lagged chromosomes at late anaphase I. c Chromosome bridges and micro nuclei at telophase I. d Micronuclei at tetrad stage. Bar $10 \mu \mathrm{m}$ 


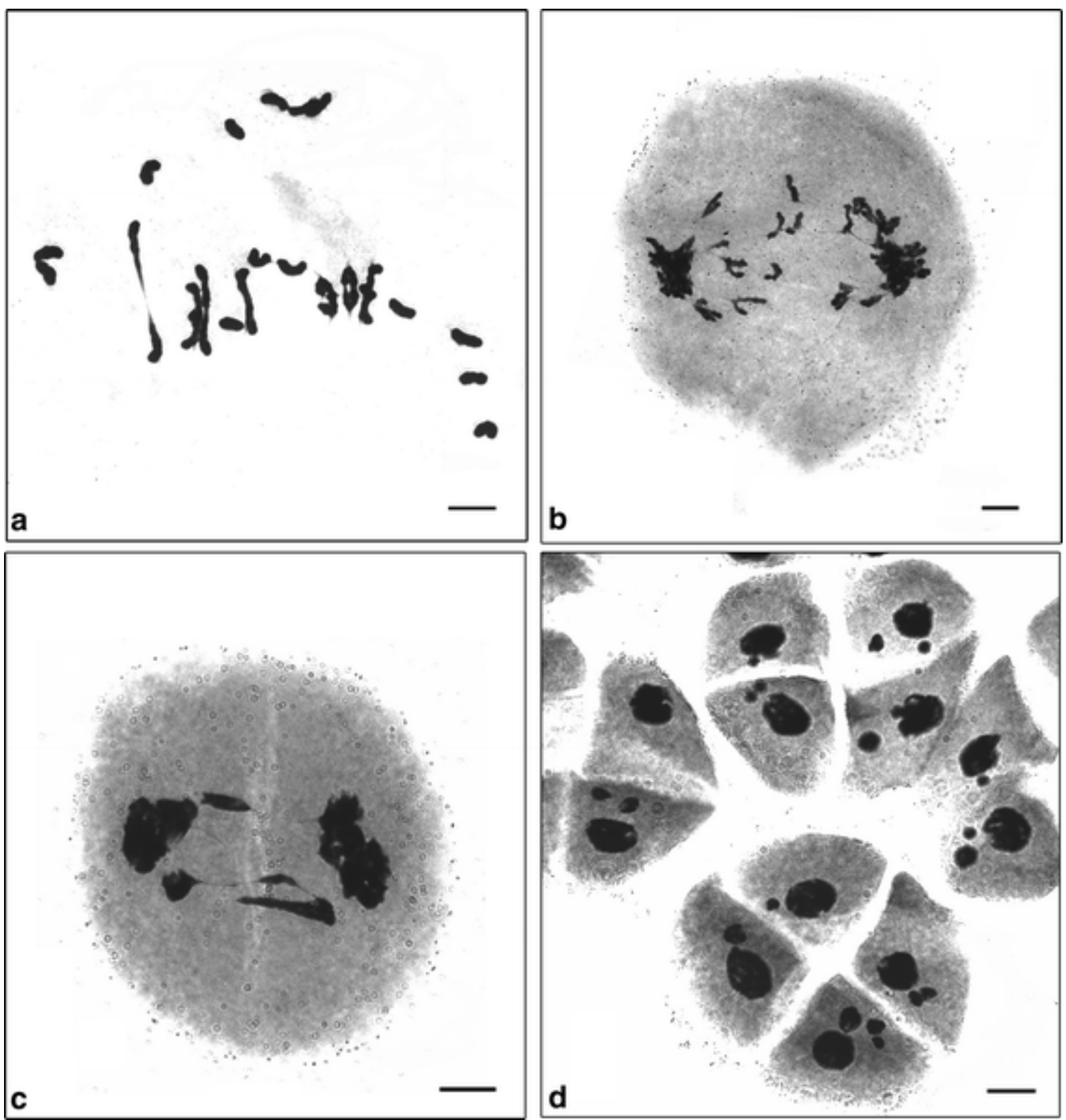

\section{Discussion}

\section{Characterization of a valuable radiation hybrid population for chromosome} 3B

A complete RH map of chromosome 3B, comprising 540 markers mapped employing 92 3B-RH lines, was initially generated (Kumar et al. 2012a). Here, the mapping information was extended to a much larger RH population (see supplementary information for details). A mini custom DArT array was developed specifically for the task. This allowed drastically reducing the cost of the analysis, thus expanding the size of the population to be genotyped. Further, the blotting of a custom array consented to increase the built-in experimental controls, and hence the final quality of the marker scoring, which is not a secondary concern when genotyping for radiation-mediated deletions.

The whole 3B-RH population was characterized at 140 loci and resulted in 2,852 cR RH map. The 
140 marker loci were evenly spaced across the chromosome at an average interval of $20 \mathrm{cR}$, calculated to correspond to $\sim 7 \mathrm{Mb}$ in physical size. The interval that separates the markers is smaller than the average size of a wheat radiation-mediated deletion, which was calculated at $26 \mathrm{Mb}$ for this 3B-RH population (Kumar et al. 2012a). This shows that the number and relative distance of the markers are sufficient to recognize the vast majority of the radiation-mediated deletions occurring on the $3 \mathrm{~B}$ chromosome, while still reducing sufficiently the experimental cost to allow genotyping of the whole 3B-RH panel.

Among the 3B-RH lines screened, 100 (14.4\%) missed the whole chromosome 3B. Typically, the $\mathrm{RH}_{1}$ lines are expected to have a single copy of chromosome $3 \mathrm{~B}$ or a portion of it. However, the existence of lines with the entire chromosome 3B missing could be explained by several reasons. First, LDN 3D (3B) was not completely male sterile. Phenotypic analysis showed $5.7 \%$ seed set and $28 \%$ PV in LDN 3D (3B) as compared to the normal LDN (Table 1). Since LDN 3D (3B) was used as female for the production of the $\mathrm{RH}_{1}$ lines, unexpected self-fertilization would inevitably result in non$\mathrm{RH}_{1}$ lines lacking the whole $3 \mathrm{~B}$ chromosome. A second explanation could be that chromosome $3 \mathrm{~B}$ is not stable in monosomic conditions, and it is possible that in rare cases the entire chromosome lags behind during cell division, producing $\mathrm{RH}_{1}$ lines completely missing the chromosome (Kynast et al. 2002). Third, some of the lines might actually result from the over damaging of the chromosome by the radiation treatment. In those cases, functional components such as the centromere or telomere might have been removed, preventing the chromosome from undergoing proper cell division (Tiwari et al. 2012). In this latter case, small portions of the $3 \mathrm{~B}$ chromosome could be translocated to other parts of the genome, too small in size to be recognized by the 140 markers employed here (Kalavacharla et al. 2006; Kumar et al. 2012a).

The characterization of the 3B-RH panel also identified 133 lines that did not show deletion for any of the 140 markers. These lines may either have escaped radiation damage to chromosome 3B, or the deletions that have been created are too small $(>7 \mathrm{Mb})$ to be identified in this study. It is in fact an established knowledge in wheat RH that the increase of markers saturation is typically accompanied by the identification of additional smaller deletions (Kalavacharla et al. 2006; Kumar et al. 2012a, $\underline{\text { b) }}$ ).

The $463 \mathrm{RH}_{1}$ lines carrying sizable deletions along the chromosome are potentially very valuable biological material for studies of 3B. Together these lines subdivide the $3 \mathrm{~B}$ chromosome into nearly 3,000 small deletion bins of approximately $350 \mathrm{~Kb}$ in size. This is a very high-map resolution for wheat, rivaled only by the $200 \mathrm{~Kb}$ resolution reached in a $\mathrm{RH}$ map of chromosome 1D (Kalavacharla et al. 2006).

\section{Environmental effect on the phenotype controlled by Tdes2}

The whole $3 \mathrm{~B}-\mathrm{RH}_{1}$ population was phenotyped using two fertility measurements ( $\mathrm{SpS}$ and PV). The broad sense heritability $\left(\mathrm{H}^{2}\right)$ was high for both traits, even though this value was calculated for chromosome $3 \mathrm{~B}$ as whole, rather than single gene(s) segregation. However, in the absence of precise experimental controlled conditions, both $\mathrm{SpS}$ and PV are known to be influenced by the environment (Elkonin and Tsvetova 2012). To minimize the experimental error caused by the environmental variability, the measurements for these traits should be replicated across different environments (Hallauer and Miranda 1988). Unfortunately, $\mathrm{RH}_{1}$ lines are unique genotypes that cannot be 
replicated, each individual characterized by a unique set of deletions. On selfing of $\mathrm{RH}_{1}$, the disomic chromosomes segregate at meiosis, unmasking the deletions that the hemizygous state was covering (carried by the chromosome derived from the irradiated LDN). Also, the monosomic chromosomes (3B and 3D in this case) would not pair at meiosis, segregating in a non-Mendelian fashion. More importantly, all the sterile $\mathrm{RH}_{1}$ lines would not produce any seeds to proceed to the following generations, thus, making it impossible to replicate the $\mathrm{RH}_{1}$ lines across environments. To provide a meaningful value of fertility from single plants measurements, multiple samples were collected for each of the two traits, and a single overall value of fertility was generated merging the two traits values. Three check genotypes with decreasing dosages of chromosome $3 \mathrm{~B}$ were phenotyped for $\mathrm{SpS}$ and $\mathrm{PV}$ in three replicates across the same environments used for testing of the $\mathrm{RH}_{1}$ lines. Non-significant interactions were detected between genotypes and environments suggesting good experimental control, while significant $(p=0.001)$ differences were observed between all three genotypes for both traits (Table 1 ). On this basis, three fertility classes were identified depending on the doses of chromosome $3 \mathrm{~B}$ (disomic, monosomic or nullisomic). The overall fertility scoring of the $3 \mathrm{~B}-\mathrm{RH}$ lines was decided in comparison to these three classes.

Interestingly, in RH the genetic variability for a given trait, when ignoring the environment influence, is due solely to the occurrence of a deletion on the gene(s) controlling the trait. The frequency at which a locus is deleted in a RH population can be measured as one minus the retention frequency. The 3B$\mathrm{RH}$ population has a retention frequency of 0.80 and hence a locus deletion frequency of 0.20 . Any 3B loci involved in the control of the fertility phenotype should then be deleted with the same frequency as any other. If one single locus is involved in the control of the fertility phenotype, then the frequency of knockout phenotypes should match the frequency of one locus deletion. This is the case of Tdes 2 , with $20 \%$ of the 3B-RH lines being sterile, matching the $20 \%$ population wise locus deletion frequency. Based on these calculations, a single locus should be involved in controlling the observed phenotype.

\section{Molecular localization of the Tdes2 locus}

QTL analysis mapped the Tdes2 locus to an interval of $38.4 \mathrm{cR}$ between the marker loci wmc326 and $w P t-8141$. These markers were located in the telomeric bin of chromosome 3BL. The estimated $\mathrm{cR}$ to $\mathrm{Mb}$ conversion ratio for deletion bin 3BL7-0.63-1.00 is $0.348 \mathrm{Mb} \mathrm{cR}^{-1}$ (Kumar et al. 2012a), so a $38.4 \mathrm{cR}$ interval corresponds to a physical size of $13 \mathrm{Mb}$. An independent confirmation of the correct positioning of the QTdes2.ndsu-3B QTL was derived by the sterility level observed for the 3BL7-0.63-1.00 deletion bin line. Further, Devos et al. (1999) found that the ditelosomic for chromosome 3BS is sterile, while Joppa and Williams (1988) reported that the long arm of chromosome 3B is necessary to maintain LDN 3D (3B) substitution line. All the evidences support our localization of the Tdes 2 locus to the telomeric or sub-telomeric portion of the long arm of chromosome $3 \mathrm{~B}$.

Marker $w P t-8983$ was found as the most strongly associated locus but explains only the $25.6 \%$ of the phenotypic variation, but precisely predicts the phenotype of $92 \%$ of the $3 \mathrm{~B}-\mathrm{RH}$ lines based on its retention/deletion scoring. There is a partial discrepancy between the relatively low $r^{2}$ value and the fact that Tdes 2 is apparently a single gene. However, the phenotypic variation in $\mathrm{RH}$ population is not due to allelic recombination and hence does not follow Mendelian genetics, meaning that the 
fertility/sterility values are not normally distributed. This might contribute to reduce the genotype to phenotype coefficient of determination. Further, there are 28 uncharted breakages between marker $w P t-8983$ and the locus governing this fertility phenotype, which correspond to a map distance of $4 \mathrm{cR}$. Based on the map to physical conversion ratio, marker wPt- 8983 is approximately $1.4 \mathrm{Mb}$ away from the functional locus. Still, the resolution of the 3B-RH map is so elevated $(\sim 350 \mathrm{~Kb})$ that even a marker less than $1.4 \mathrm{Mb}$ away from the gene controlling the function is predictive for just the $25.6 \%$ of the phenotypic variation. In addition, there are 32 lines that lack the entire $3 \mathrm{~B}$ chromosome but still show a fertile phenotype, likely carrying a very small translocated portion containing Tdes 2 . Further, 15 lines with no identified deletions show a sterile phenotype, probably because they harbor a small undetected deletion within the Tdes 2 region. Other explanations for the observed phenotypes to genotype discrepancies are also plausible, but additional markers saturation and detailed characterization of these 75 informative lines (commonly defined "recombinants" in genetic mapping studies) will hopefully provide a better understanding of their behavior and ultimately prove effective to precisely pinpoint the location of the Tdes2 gene.

\section{Wheat lines defective of the Tdes 2 locus resemble maize mutants for $d y$, a syntenic locus controlling synapsis at meiosis}

The preliminary data gathered here provide novel and interesting information about the biological function of the Tdes2 gene. Probably, the most surprising piece of evidence is the fact that a dosage effect was observed for this gene. Lines hemizygous for the locus, as represented by the double monosomic $\mathrm{F}_{1}$, suffer partial sterility. This indicates that a copy on each $3 \mathrm{~B}$ sister chromosomes is required for proper meiosis. Further, the DNA undergoes re-synthesis and duplication during interphase, meaning that in a hemizygous individual one copy would be duplicated to two, instead of the duplication from two to four that occurs in a homozygous line. It is unclear whether all of the resynthesised copies are actually expressed during meiotic division, but if that was the case the lack of one copy would maximize its effect, changing from four expressed loci to just two. Also, only two of the four forming gametophytes will receive a copy of Tdes 2 at the end of meiosis. While this would explain the nearly $50 \%$ viability observed (i.e. only $50 \%$ of the gametophytes have Tdes2), it does not explain why the most drastic effects are observed at diakinesis/metaphase during the first meiotic division, while the single meiotic cell still carries all available copies of Tdes2. It is then likely that the observed $50 \%$ fertility in hemizygous plants is just coincidental, and not the direct result of a reduction in the copy number of Tdes 2 . Interestingly, a desynaptic $(d y)$ mutation in maize located on its chromosome 3 was also shown to have a dosage effect on the overall plant fertility (Murphy and Bass 2012).

Previous studies in wheat have proposed that the removal of chromosome 3B results in desynapsis during meiosis (Sears 1954; Joppa and Williams 1988; Devos et al. 1999). In the past, the term desynapsis was used in a loose sense, meaning any mutation that ultimately resulted in the formation of univalents at diakinesis (Bourne and Danielli 1979). As the scientific knowledge has progressed over the years, the different aspects of meiosis have been better understood. Today, the term "de-synapsis" (or a-synapsis) is mainly employed in its historical sense to refer to mutants that were described in the past, while in modern meiosis literature preference is typically given to those terms that more precisely describe the molecular function during meiosis (Pawlowski et al. 2003). Sears (1954) has defined this mutation as "desynaptic" in times that preceded the clear understanding of the synaptonemal complex 
formation, and all authors that followed employed his definition. It seems then inappropriate to change the name today. However, it has to be kept in mind that the Tdes2 gene might not be causing desynapsis in senso stricto, which refers to the failure of properly forming the synaptonemal complex. In fact, it is yet unclear if the removal of the Tdes 2 locus affects the formation of the synaptonemal complex per se. What this study confirms is that the following stage(s) of meiosis are certainly affected, during which the synaptonemal complex is degraded and the bivalents are kept together by the chiasmata.

The surge of univalents at diakinesis/metaphase and of micronuclei in the tetrad of pollen mother cells (Fig. 6) are common features of desynaptic mutants, also shared with the $d y$ maize mutants (Murphy and Bass 2012). Further, the $d y$ mutation was shown to cause desynapsis by disrupting the normal clustering of telomeres during bouquet formation, which ultimately affects the proper progress through pachytene (Murphy and Bass 2012). This is of particular importance considering the fact that chromosome 3L of maize that harbors $d y$ shares conserved synteny with chromosomes 3 of Sorghum bicholor L. (Paterson et al. 2009), 1 of rice (International Rice Genome Sequencing Project 2005), 2 of Brachypodium distachyon (The International Brachypodium Initiative 2010), and especially 3BL of wheat (Rustenholz et al. 2011; Brenchley et al. 2012; Bolot et al. 2009). The similarity of effects between $d y$ and $\Delta T d e s 2$ and their syntenic relationships suggest that these could be orthologus genes, probably originated from a common ancestral gene that remained conserved throughout the evolution of the grasses.

The map location of the $d y$ locus was narrowed down to a $9 \mathrm{cM}$ (approximately $7.7 \mathrm{Mb}$ ) interval of the maize genetic bin 3.06 by means of B-A translocation mapping (Murphy and Bass 2012). The genome of wheat is yet to become entirely available (Brenchley et al. 2012), but by molecular estimation, the Tdes 2 locus was pin pointed to a $4 \mathrm{cR}$ (approximately $1.4 \mathrm{Mb}$ ) interval of the deletion bin 3BL7 by means of RH mapping. By comparison, both approaches proved successful in identifying the genomic region harboring the gene of interest. However, the interval identified through $\mathrm{RH}$ mapping is sixfold more refined in a genome sevenfold larger (Schnable et al. 2009; Brenchley et al. 2012). The parallel study of both orthologus genes will ultimately help in better characterizing the molecular mechanisms that drive desynapsis in cereals. In this regard, the complete sequence of at least one of the two genes (Tdes2 or $d y$ ) is an indispensable requirement to follow their molecular behavior during meiosis. The study presented here is the first step toward the precise dissection of the Tdes2 locus.

\section{Conclusions}

The chromosomal location of the Tdes 2 gene has been known for nearly 60 years, but its lack of genetic diversity has precluded any attempt to fine map this locus. Here, the use of QTL analysis on $\mathrm{RH}$ allowed localizing the elusive Tdes 2 to a region of just few $\mathrm{Mb}$ in size. Radiation hybrids QTL analysis represents a novel approach that holds great potential to map all those genes for which no genetic diversity is currently known. Often these types of genes control those life-depending cellular functions so dear to biologists, but are difficult to study because of their lack of natural variation. In this regard, RH functional mapping provides a good experimental solution to a difficult challenge.

The biological function of Tdes 2 remains unclear, but the data presented here allowed for a first 
glimpse to its involvement in wheat meiosis. The practical applications of this gene are many and significant. Its involvement in control of bivalents formation at diakinesis and its confirmed dosage effect raise the question of whether a number of copies higher than two could further promote pairing/recombination to benefit selection in recombination-based breeding. Also, the silencing of this gene provides a nuclear system to produce male sterile (female) plants, the first fundamental step toward commercial production of wheat hybrids. Moreover, from an academic prospective, the unraveling of the function of this gene would help in understanding the complex mechanism that regulates meiosis in polyploid species, arguably one of the most important aspects that led to the evolution of wheat and other polyploid crops. Still, in order to achieve all of these goals, the complete sequence of this gene has to be obtained by means of map-based cloning. The QTL analysis and the $\mathrm{RH}$ population resources presented here are only the first step in the difficult path toward the cloning of the Tdes 2 locus, but are at least a first step in the right direction.

\section{Acknowledgments}

The authors wish to thank Professor Wojtek Pawlowski (Cornell University, NY) for in-depth revision and insightful comments on this manuscript, Justin Hegstad and Allen Peckrul for their qualified technical help. This work was supported by funding from the National Science Foundation, Plant Genome Research Program (NSF-PGRP) grant No. IOS-0822100 to SFK. F.M.B was partially supported by Program Master and Back Regione Autonoma della Sardegna and Monsanto Beachell-Borlaug International Scholarship.

\section{Electronic supplementary material}

Below is the link to the electronic supplementary material.

Supplementary material 1 (DOCX $640 \mathrm{~kb}$ )

\section{References}

Akbari M, Wenzl P, Caig V et al (2006) Diversity arrays technology (DArT) for high-throughput profiling of the hexaploid wheat genome. Theor Appl Genet 113:1409-1420

Al-Kaff N, Knight E, Bertin I, Foote T, Hart N, Griffiths S, Moore G (2008) Detailed dissection of the chromosomal region containing the $P h 1$ locus in wheat Triticum aestivum: with deletion mutants and expression profiling. Ann Bot $101: 863-872$

Baskaran R (2010) Emerging role of radiation induced bystander effects: cell communications and carcinogenesis. Genome Integr 1:13

Beadle GW (1930) Genetic and cytological studies of a Mendelian asynaptic in Zea mays. Cornell Agric Exp Stn Mem 129:1-23

Bolot S, Abrouk M, Masood-Quraishi U et al (2009) The 'inner circle' of the 
cereal genomes. Curr Opin Plant Biol 12:119-125

Bourne GH, Danielli JF (1979) International review of cytology, vol 58. Academic Press Inc, London

Brenchley R, Spannagl M, Pfeifer M et al (2012) Analysis of the bread wheat genome using whole-genome shotgun sequencing. Nature 491(7426):705-710

Cande ZW, Freeling M (2011) Inna Golubovskaya: the life of a geneticist studying meiosis. Genetics 188(3):491-498

Caryl AP, Gareth HJ, Franklin FCH (2003) Dissecting plant meiosis using Arabidopsis thaliana mutants. J Exp Botany 54(380):25-38

Churchill GA, Doerge RW (1994) Empirical threshold values for quantitative trait mapping. Genetics 138:963-971

Cox DR, Burmeister M, Price ER, Kim S, Myers RM (1990) Radiation hybrid mapping: a somatic cell genetic method for construction of high-resolution maps of mammalian chromosomes. Science 250:245-250

de Boer E, Heyting C (2006) The diverse roles of transverse filaments of synaptonemal complexes in meiosis. Chromosoma 115:220-234

de Givry S, Bouchez M, Chabrier P, Milan D, Schiex T (2005) Carthagene: multi population integrated genetic and radiated hybrid mapping. Bioinformatics 21:1703-1704

Devos KM, Sorrells ME, Anderson JA et al (1999) Chromosome aberrations in wheat nullisomic-tetrasomic and ditelosomic lines. Cereal Res Commun 27(3):231-239

Dolezel J, Simkova H, Kubalakova M et al (2009) Chromosome genomics in the Triticeae. In: Feuillet C, Muehlbauer GJ (eds) Genetics and genomics of the Triticeae. Springer, New York, pp 285-316

Elkonin LA, Tsvetova MI (2012) Heritable effect of plant water availability conditions on restoration of male fertility in the "9E" CMS-inducing cytoplasm of sorghum. Front Plant Sci 3:91

Endo TR, Gill BS (1996) The deletion stock of common wheat. J Hered 87(4):295-307

Falconer DS, Mackay TFC (1996) Introduction to quantitative genetics, 4th edn. Addison Wesley Longman Limited, Edinburgh Gate

Faraut T (2009) Contribution of radiation hybrids to genome mapping in domestic animals. Cytogenet Genome Res 126:21-33 
Faris JD, Gill BS (2003) Genomic targeting and high-resolution mapping of the domestication gene $Q$ in wheat. Genome 45:706-719

Franckowiak JD, Lundqvist U (2008) Table of barley genetic stock description. Barley Genetics Newsl 38:134-164

Gill BS, Friebe B, Endo TR (1991) Standard karyotype and nomenclature system for description of chromosome bands and structural aberrations in wheat (Triticum aestivum). Genome 34:830-839

Gill KS, Gill BS, Endo TR, Boyko EV (1996) Identification and high-density mapping of gene-rich regions in chromosome group 5 of wheat. Genetics $143: 1001-1012$

Goss SJ, Harris H (1975) New method for mapping genes in human chromosomes. Nature 255:680-684

Guidet F, Rogowsky P, Taylor C, Song W, Langridge P (1991) Cloning and characterisation of a new rye-specific repeated sequence. Genome 34:81-87

Hallauer AR, Miranda JB (1988) Quantitative research in maize breeding. Iowa State University Press, Ames

Hassold T, Sherman S, Hunt P (2009) Counting cross-overs: characterizing meiotic recombination in mammals. Hum Mol Genet 9(16):2409-2419

Henderson AS (1970) The time and place of meiotic crossing-over. Annu Rev Genet 4:295-324

Hernandez-Soriano JM, Ramage RT (1973) Desynaptic genes. Barley Genetics News1 4:123-125

Higgins JD, Sanchez-Moran E, Armstrong SJ, Jones GH, Franklin FC (2005) The Arabidopsis synaptonemal complex protein ZYP1 is required for chromosome synapsis and normal fidelity of crossing over. Genes Dev 19(20):2488-2500

Hossain KG, Riera-Lizarazu O, Kalavacharla V, Vales MI, Maan SS, Kianian SF (2004) Radiation hybrid mapping of the species cytoplasm-specific (scs ${ }^{a e}$ ) gene in wheat. Genetics 168:415-423

Huang BE, George AW, Forrest KL, Kilian A, Hayden MJ, Morell MK, Cavanagh CR (2012) A multiparent advanced generation inter-cross population for genetic analysis in wheat. Plant Biotechnol J 10:826-839

International Rice Genome Sequencing Project (2005) The map-based sequence of the rice genome. Nature 436:793-800

Joppa LR, Williams ND (1977) D-genome substitution monosomics of durum 
wheat. Crop Sci 17:772-776

Joppa LR, Williams ND (1988) Langdon durum disomic substitution lines and aneuploid analysis in tetraploid wheat. Genome 30:222-228

Joppa LR, Williams ND (1993) The Langdon durum disomic-substitutions: development, characteristics, and uses. Agron Abstr, p 68

Kalavacharla V, Hossain K, Gu Y et al (2006) High-resolution radiation hybrid map of wheat chromosome 1D. Genetics 173:1089-1099

Kim N (2001) Disseminating the genome: joining, resolving, and separating sister chromatids during mitosis and meiosis. Annu Rev Genet 35:673-745

Kumar A, Bassi FM, Paux E et al (2012a) DNA repair and crossing over favor similar chromosome regions as discovered in radiation hybrids of Triticum. BMC Genomics 13:339

Kumar A, Simons K, Iqbal M et al (2012b) Physical mapping resources for large plant genomes: radiation hybrids for wheat D-genome progenitor Aegilops tauschii. BMC Genomics 13:597

Kynast RG, Okagaki RJ, Rines HW, Phillips RL (2002) Maize individualized chromosome and derived radiation hybrid lines and their use in functional genomics. Funct Integr Genomic 2:60-69

Li HW, Pao WK, Li CH (1945) Desynapsis in the common wheat. Am J Bot 32(2):92-101

Martini G, Bozzini A (1966) Radiation induced asynaptic muattions in durum wheat (Triticum durum Desf.). Chromosoma 20:251-266

McIntosh RA, Dubcovsky J, Rogers WJ, Morris C, Apples R, Xia XC (2011) Catalogue of gene symbols for wheat: 2011 supplement. Annu Wheat Newsl 57

Michalak de Jimenez MK, Bassi FM, Ghavami F et al (2013) A radiation hybrid map of chromosome 1D reveals synteny conservation at a wheat speciation locus. Funct Integr Genomics 13:19-32

Murphy SP, Bass HW (2012) The maize (Zea mays) desynaptic (dy) mutation defines a pathway for meiotic chromosome segregation, linking nuclear morphology, telomere distribution and synapsis. J Cell Sci 125(15):3681-3690

Paterson AH, Bowers JE, Bruggmann R et al (2009) The Sorghum bicolor genome and the diversification of grasses. Nature 457:551-556

Paux E, Roger D, Badaeva E, Gay G, Bernard M, Sourdille P, Feuillet C (2006) Characterizing the composition and evolution of homoeologous genomes in 
hexaploid wheat through BAC-end sequencing on chromosome 3B. Plant J $48: 463-474$

Paux E, Sourdille P, Salse J et al (2008) A physical map of the 1-gigabase bread wheat chromosome 3B. Science 322:101-104

Paux E, Faure S, Choulet F et al (2010) Insertion site-based polymorphism markers open new perspectives for genome saturation and marker-assisted selection in wheat. Plant Biotechnol J 8:196-210

Pawlowski WP, Golubovskaya IN, Cande WZ (2003) Altered nuclear distribution of recombination protein RAD51 in maize mutants suggests the involvement of RAD51 in meiotic homology recognition. Plant Cell 15:1807-1816

Peterson R, Slovin JP, Chen C (2010) A simplified method for differential staining of aborted and non-aborted pollen grains. Int J Plant Biol 1:e13

Qiao H, Chen JK, Reynolds A, Hoog C, Paddy M, Hunter N (2012) Interplay between synaptonemal complex, homologous recombination, and centromeres during mammalian meiosis. PLoS Genet 8(6):e1002790

Raman H, Zhang K, Cakir M et al (2005) Molecular characterization and mapping of ALMT1, the aluminium-tolerance gene of bread wheat (Triticum aestivum L.). Genome 8:781-791

Ross KJ, Fransz P, Jones GH (1996) A light microscope atlas of meiosis in Arabidopsis thaliana. Chromosom Res 4:507-516

Rustenholz C, Choulet F, Laugier C et al (2011) A 3,000-loci transcript map of chromosome $3 \mathrm{~B}$ unravels the structural and functional features of gene islands in hexaploid wheat. Plant Physiol 157:1596-1608

Schnable PS, Ware D, Fulton RS et al (2009) The B73 maize genome: complexity, diversity, dynamics. Science 326:1112-1115

Schramm S, Fraune J, Naumann R et al (2011) A novel mouse synaptonemal complex protein is essential for loading of central element proteins, recombination, and fertility. PLoS Genet 7(5):e1002088

Sears ER (1954) The aneuploids of common wheat. Missouri Agr Exp Stn Res Bull $572: 1-58$

Somers DJ, Isaac P, Edwards K (2004) A high-density microsatellite consensus map for bread wheat (Triticum aestivum L.). Theor Appl Genet 109:1 1051114

Song QJ, Shi JR, Singh S et al (2005) Development and mapping of microsatellite (SSR) markers in wheat. Theor Appl Genet 110:550-560 
Sutka J, Galiba G, Vagujfalvi A, Gill BS, Snape JW (1999) Physical mapping of $V r n-A 1$ and genes on chromosome 5A of wheat using deletion line. Theor Appl Genet 99:199-202

Tanaka A, Shikazono N, Hase Y (2010) Studies on biological effects of ion beams on lethality, molecular nature of mutation, mutation rate, and spectrum of mutation phenotype for mutation breeding in higher plants. J Radiat Res 51:223233

The International Brachypodium Initiative (2010) Genome sequencing and analysis of the model grass Brachypodium distachyon. Nature 463:763-768

Tiwari VK, Riera-Lizarazu O, Gunn HL et al (2012) Endosperm tolerance of paternal aneuploidy allows radiation-hybrid mapping of the wheat D-genome and a measure of $\gamma$ ray-induced chromosome breaks. PLoS ONE 7(11):e48815

Tsilo TJ, Hareland GA, Chao S, Anderson JA (2011) Genetic mapping and QTL analysis of flour color and milling yield related traits using recombinant inbred lines in hard red spring wheat. Crop Sci 51:237-246

Wang S, Basten CJ, Zeng ZB (2011) Windows QTL Cartographer 2.5. Department of Statistics, North Carolina State University, Raleigh

Watanabe N, Koval SF (2003) Mapping of chlorine mutant genes on the long arm of homoeologous group 7 chromosomes in common wheat with partial deletion lines. Euphytica 129:259-265

Wenzl P, Suchánková P, Carling J et al (2010) Isolated chromosomes as a new and efficient source of DArT markers for the saturation of genetic maps. Theor Appl Genet 121:465-474

Xu SJ, Joppa LR (1995) Mechanisms and inheritance of first division restitution in hybrids of wheat, rye, and Aegilops squarrosa. Genome 38:607-615

Xu SJ, Joppa LR (2000) Hexaploid triticales from hybrids of Langdon durum Dgenome substitutions with rye. Plant Breed 119:223-226

Zhirov EG, Bessarab KS, Gubanova MA (1974) Genetic studies on partial desynapsis in soft wheat. Soviet Genetics 9(1):10-18

Zhou C, Dong W, Han L et al (2012) Construction of whole genome radiation hybrid panels and map of chromosome $5 \mathrm{~A}$ of wheat using asymmetric somatic hybridization. PLoS ONE 7(7):e40214 
